\title{
Editorial introduction to the special issue
}

\author{
Salem Benferhat
}

Published online: 4 May 2013

(C) Springer Science+Business Media Dordrecht 2013

The information available to intelligent agents is often uncertain, inconsistent and incomplete. It is then crucially important to develop logics for uncertainty to achieve different complex reasoning tasks. Uncertainty is present in many applications and may be represented using different frameworks: plausibility orderings, probability theory frameworks, argumentation systems, etc.

This special issue gathers five contributions that cover different aspects of reasoning under uncertainty using non classical logics. Four of the five papers are fully revised and extended versions of contributions initially presented at the Fifth International Conference on Scalable Uncertainty Management (SUM'11)

The two first papers concern extensions of logic-based programming languages to deal with uncertain information.

The paper by Pere Pardo, Lluìs Godo entitled "t-DeLP: an argumentation-based Temporal Defeasible Logic Programming framework" deals with handling temporal information using defeasible logic. The proposed framework, called t-DeLP, allows to extend argumentation-based defeasible logic in order to represent temporal processes. The idea is to associate temporal information to literals and to define temporal logic programs are sets of basic temporal facts and (strict or defeasible) durative rules. Concepts of argumentation systems, such as the one of undefeated arguments, are redefined in this t-DeLP framework. A procedure for determining a set of warranted literals is proposed. The paper also discusses rational properties satisfied by the t-DeLP framework.

The paper by Georg Gottlob,Thomas Lukasiewicz, Maria Vanina Martinez and Gerardo I. Simari entitled "Query Answering under Probabilistic Uncertainty in Datalog+/ Ontologies" concerns an extension of Datalog +/- to deal with uncertainty. The authors use Markov Logic Networks to provide a probabilistic semantics for the probabilistic Datalog+/-. They provide scalable and sound algorithms for

S. Benferhat $(\bowtie)$

CRIL-CNRS, Université d'Artois, Faculté Jean Perrin. Rue Jean Souvraz,

SP 18, 62307 Lens, France

e-mail: benferhat@cril.fr 
answering threshold queries of the form "what is the set of all ground atoms that are inferred from a given probabilistic ontology with a probability of at least $\mathrm{p}$ ?". They also analyse the problem of inconsistency that can arise in probabilistic Datalog $+/-$. They propose two different approaches for computing preferred repairs and study their associated computational complexity.

The third paper by Sébastien Konieczny and Ramon Pino Pérez entitled "Confluence operators and their relationships with Revision, Update and Merging?" deals with dynamics of knowledge. It first reviews different forms of belief changes and gives some relationships between revision, update and merging operators. Then the authors introduce general belief change operators called confluence operators. These operators are to merging operators what update operators are to revision operators. The authors establish then a representation theorem for confluence operators and show links between confluence and update and merging operators. Lastly, a family of confluence operators based on distances are defined.

The two last papers propose two different approaches to tackle the hard problem of computing accepted arguments in argumentation systems. Argumentation is an important model for reasoning from uncertain and inconsistent pieces of information.

The paper by Sebastian Gottifredi, Nicolás D. Rotstein, Alejandro J. García and Guillermo R. Simari entitled "Using Argument Strength for Building Dialectical Bonsai" deals with the problem of computing accepted arguments through dialectical trees. Given an argumentation system, in order to check whether an argument is accepted or not, a tree of arguments rooted in it is built and evaluated. Such argumentation trees may be very large. The paper proposes a heuristics-based pruning technique over argumentation trees. The heuristic is based on a measure of strength, assigned to arguments, computed using dynamic programming techniques. The authors use dynamic argumentation system as a framework for illustrating their approach. They provide empirical testing showing that, in most cases, using heuristics answers queries much faster than the usual techniques. Lastly, a set of postulates characterising the desired behaviour of any strength formula has been proposed.

The paper by Leila Amgoud and Caroline Devred entitled "Argumentation frameworks as Constraint Satisfaction Problems" deals with the problem of reasoning from argumentation systems. Different semantics have been proposed to evaluate the strength or acceptability of arguments. The paper studies how to encode the problem of computing the extensions of an argumentation framework as a constraint satisfaction problem (CSP) for several argumentation systems. This encoding is important since it makes it possible to use very efficient CSP solvers for computing the extensions. The paper also analyses the encoding, using CSP, of two other families of argumentation frameworks: the constrained version of Dung's abstract framework and preference-based argumentation framework which is very appropriate when one deals with uncertainty.

Acknowledgements The editor is particularly indebted to all the colleagues who helped him at various stages of the reviewing process. 